\title{
Conidiophore Stalk-less1 Encodes a Putative Zinc-Finger Protein Involved in the Early Stage of Conidiation and Mycelial Infection in Magnaporthe oryzae
}

\author{
Zhuangzhi Zhou, ${ }^{1,2}$ Guihua Li, ${ }^{1,2}$ Chunhua Lin, ${ }^{1}$ and Chaozu He${ }^{1}$ \\ ${ }^{1}$ State Key Laboratory of Plant Genomics, Institute of Microbiology, Chinese Academy of Sciences, Beijing 100101, P. R. \\ China; ${ }^{2}$ Graduate School of the Chinese Academy of Sciences, Beijing 100039, P. R. China
}

Submitted 24 April 2008. Accepted 14 December 2008.

\begin{abstract}
Over recent decades, many pathogenicity genes of Magnaporthe oryzae have been identified but only a very limited number of genes have been identified that encode components of the conidiogenesis pathway. We report here a $\mathrm{T}$ DNA insertional mutant that completely lost conidiation ability. Further investigation revealed that this mutant did not develop any conidiophore, and that the T-DNA was integrated into an annotated gene designated as conidiophore stalk-less 1 or COS1. Complementation experiments suggested that $C O S 1$ may be a determinant of conidiation. Sequence analysis revealed that $C O S 1$ putatively encodes a 491-amino-acid zinc-finger protein and the protein was revealed localized to nucleus. Reverse-transcriptase polymerase chain reaction (RT-PCR)-based expression analysis indicated that two homologues of conidiophore-related genes were affected by the $\cos 1$ mutation, suggesting that Cos1 may function as a transcriptional regulator controlling genes responsible for conidiation. Inoculations of rice roots and wounded leaves with mycelia suggested that COS1 is not required for pathogenicity. Moreover, mutation of COS1 may aggravate infection of wounded leaves. Interestingly, different from the wild-type strain, mycelia of the $\cos 1$ mutant successfully infected host cells and caused visible symptoms on unwounded leaf blades and sheaths, indicating that Cos1 may have a role in some unknown mechanism of mycelial infection of M. oryzae.
\end{abstract}

\footnotetext{
Magnaporthe oryzae is the causal agent of rice blast disease, the most devastating phytopathogen of rice worldwide and a model organism for fungal biology (Barr 1977; Dean et al. 2005; Valent and Chumley 1991). Asexual spores (conidia) of the filamentous ascomycete fungus are the major inoculum of rice blast disease. During conidiogenesis, the fungus produces holoblastic-type conidia that result from "blowing-out" of conidiophores. The latter are specialized hyphae that are usually stalked, lifting the conidia off the substrate for better dispersal. A conidiophore usually bears three to five conidia, or more. A mature conidium is a three-celled, pyriform structure. Conidia
}

Corresponding author: Chaozu He; Telephone number: +86-10-64807397; Fax: +86-10-64858245; E-mail: hecz@im.ac.cn

* The $\boldsymbol{e}$-Xtra logo stands for "electronic extra" and indicates that Figures 7 and 8 appear in color online. Two supplemental figures are also published online. are successively and sympodially produced on each conidiophore (Howard 1994).

Blast disease occurs when a conidium attaches to a susceptible rice leaf. The conidium germinates and then develops a structure known as an appressorium. Turgor pressure in the appressorium can reach $8 \mathrm{mPa}$, which provides sufficient force to pierce the tough leaf cuticle (Talbot 2003). Subsequent colonization of host cells results in a necrotic spot on the leaf, which may produce abundant conidia that can trigger the next infection cycle. It has been reported that the severity of disease is proportional to the amount of colonization of plant tissue and the quantity of spores produced in the lesion (Teng et al. 1991). Therefore, sporulation plays a key role in the infection cycle of the fungal pathogen. Blocking conidiogenesis in its early stages is epidemiologically the most efficient strategy for disease management.

Over recent decades, great progress has been made in identifying genes encoding products that contribute to pathogenicity of M. oryzae (Talbot 2003). However, only a limited number of genes have been identified that encode components of the conidiogenesis pathway. The first morphogenetic locus that was discovered was named smo (for spore morphology). Mutation of that locus produced diverse shapes of conidia but the mutant sporulated normally (Hamer et al. 1989). Shi and Leung (1994, 1995) also defined several genetic loci controlling various stages of conidiogenesis; however, to date, most of those loci have not been characterized. The acropetal gene is believed to encode a stage-specific negative regulator of conidiation that is required to establish a sympodial pattern of spore formation. A non-sense mutation of acropetal causes indeterminate growth of the conidial tip cell that results in the production of head-totail arrays of spores (Lau and Hamer 1998). The CON7 gene, one of the morphogenetic loci defined by Shi and Leung, was characterized recently (Odenbach et al. 2007). This gene encodes a transcription factor required for the transcription of several genes that participate in disease-related morphogenesis. Though a modest but reproducible reduction in conidiation was described for the con $7^{-}$mutant, the roles of Con7p in regulation of conidiation were not addressed (Odenbach et al. 2007). It is noteworthy that Rgs 1 was recently characterized as a negative regulator of conidiation (Liu et al. 2007).

Shi and associates (1998) proposed a model describing epistatic and interactive relationships among morphogenetic loci in conidiogenesis; however, the majority of genes that determine the progression of conidial development in M. oryzae remain unknown at this time. 
A central regulatory pathway exists for the conidiation of Aspergillus nidulans, the key constituents being $\operatorname{brlA}$, abaA, and wetA (Adams et al. 1998). These genes control conidiation-specific gene expression and determine the order of gene activation during conidiophore development and spore maturation. Many developmental regulator-encoding genes, including "fluffy" genes such as $f l u G, f l b E, f l b D, f l b B, f l b C$, and $f l b A$, have been characterized in this pathway. A series of conidiation-specific con genes have also been characterized for the mold Neurospora crassa (Springer 1993), which increases our knowledge about the sporulation pathway of the fungus.

To elucidate the regulatory pathway of conidiation for $M$. oryzae, we characterized a novel zinc-finger protein-encoding gene, conidiophore stalk-less 1 (COS1). T-DNA insertional mutation of COS1 resulted in developmental failure of conidiophores. Cos 1 may function as a transcriptional regulator controlling genes that are responsible for conidiation. Interestingly, mycelia of the cos 1 mutant can infect host cells and cause visible symptoms on unwounded leaf blades, whereas mycelia of the wild type cannot.

\section{RESULTS}

\section{Identification of a conidiophore stalk-less mutant} of M. oryzae.

We previously established a T-DNA insertional library of $M$. oryzae containing 6,179 transformants by Agrobacterium tumefaciens-mediated transformation ( $\mathrm{Li}$ et al. 2007). The $\mathrm{T}$ DNA vector used was $\mathrm{pBHt} 2$, which harbors the hygromycin phosphotransferase gene ( $h p t$ ) under the control of the Aspergillus nidulans trpC promoter (Mullins et al. 2001). After phenotypic screening, we identified a conidia-deficient mutant strain M2942. Though M2942 displayed a vegetative growth rate and colony morphology similar to the wild type when grown on oatmeal agar plates (Fig. 1A), no conidia were found among aerial cultures. Light microscopic examination of the aerial cultures stained with lactophenol cotton blue revealed that M2942 did not develop conidiophore stalks (Fig. 1B). This result was consistent with the observation from scanning electron microscopy, showing that no conidiophore-like structures existed in the culture of the mutant. These observations
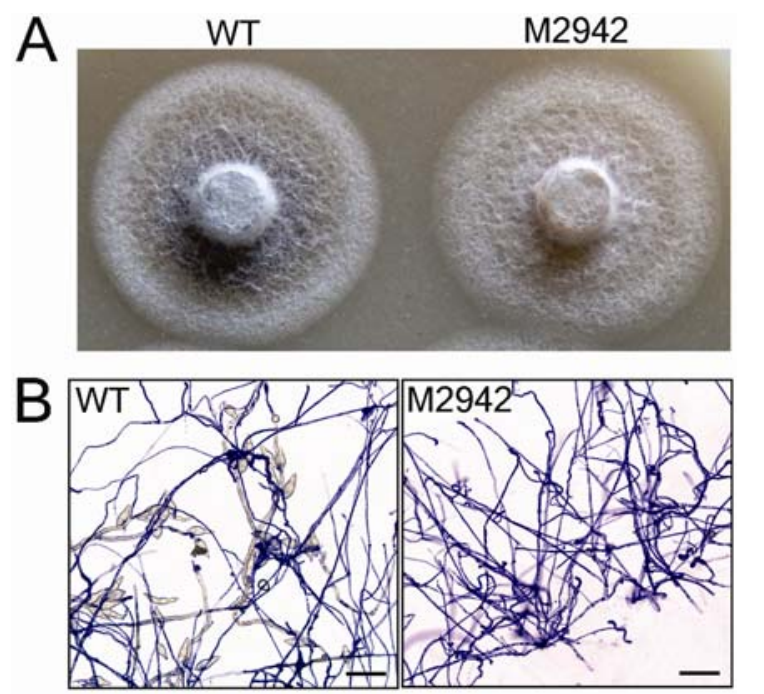

Fig. 1. Phenotypes of mutant M2942 and the wild-type strain Y34 (WT). A, Colony morphology of M2942 and the WT grown on oatmeal agar plates for 5 days. B, Aerial cultures stained with lactophenol cotton blue and observed under light microscope. Hyphae were stained blue, whereas conidiophore stalks and conidia were in gray. Bar $=20 \mu \mathrm{m}$. suggested that the conidial defect of M2942 was due to the developmental failure of the conidiophore stalk.

Southern blotting analysis indicated that a single copy of TDNA integrated into the genome of M2942 (data not shown). The flanking sequence of the T-DNA right border was obtained by a thermal asymmetric interlaced polymerase chain reaction (TAIL-PCR), which revealed that the T-DNA was integrated into an annotated gene, $M G G \_03977.5$. The insertion was confirmed by PCR using a pair of primers that spanned the insertional site (Fig. 2A). Further sequence analysis demonstrated that the TDNA was inserted into $M G G \_03977.5$ without causing any DNA deletion or duplication (Fig. 2B). The exact insertion site was at position 1373947 within supercontig 5.187. We designated the annotated gene as conidiophore stalk-less1 or COS1.

\section{A full length of $\operatorname{COS} 1$ gene restores sporulation of the mutant.}

To determine the COS1 gene's function, a plasmid harboring the full-length COS1 gene and a sulfonylurea-resistant gene $(S U R)$ as a selectable marker was introduced into mycelia of M2942. Four sulfonylurea-resistant transformants were obtained. Southern blotting assays indicated that, in one of the transformants, CM2942-1, the complemented construct was integrated into the genome of M2942 (Fig. 3A and B). CM2942-1 displayed normal sporulation. Additionally, in reverse-transcriptase (RT)-PCR analysis, COS1 transcripts were detected in CM2942-1 and the wild type while they were absent in M2942 (Fig. 3C). These results indicated that COS1 may be a determinant of sporulation in $M$. oryzae.

\section{COS1 encodes a novel zinc-finger protein localized to nucleus.}

We obtained the coding sequence of COS1 gene via RTPCR. This gene putatively encodes a 491-amino-acid protein, which is identical to a gene in GenBank (ABB89847). Sequence analysis indicated that Cos1 contains four multipleadjacent $\mathrm{C}_{2} \mathrm{H}_{2}$-type zinc-finger domains, which can potentially bind to DNA, RNA, or protein (Iuchi 2001). The motifs are most homologous to the zinc-finger protein Azf1p of Saccharomyces cerevisiae (Stein et al. 1998), with $43 \%$ (48/110)

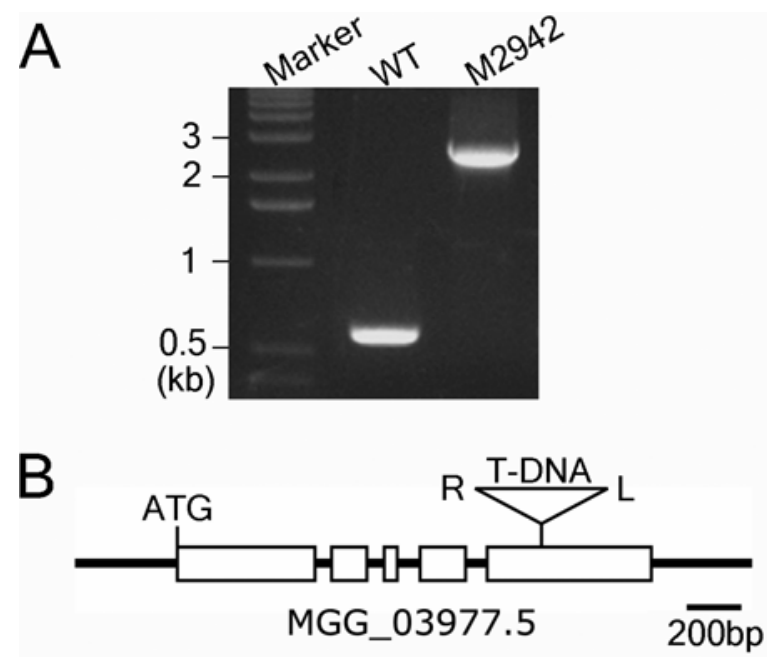

Fig. 2. T-DNA insertion event in the mutant strain M2942. A, Polymerase chain reaction amplification using paired primers RT2942F and RT2942R. The amplified product of M2942 was approximately $2.2 \mathrm{~kb}$, a size consistent with the T-DNA length, longer than that on the wild type (WT). B, Schematic diagram showing T-DNA integrated into MGG_03977.5 (COS1) gene. Rectangles are for exons of the gene and ATG, R, and L correspond to the transcriptional start code, T-DNA right border, and left border, respectively. 
identity and 58\% (64/110) similarity. Except in the motif regions, Cos 1 does not show any significant homology with other known proteins, suggesting that it is a novel zinc-finger protein.

To specify the subcellular localization of Cos1, we fused the coding sequence of COS1 at its $3^{\prime}$ terminus with a green fluorescent protein (GFP) gene and introduced the construct into the M2942 strain. The fusion protein was expressed under the control of the $\operatorname{trp} C$ promoter. G3-1, a derivative transformant of COS1-GFP, restored sporulation of M2942, indicating that the Cos1-GFP fusion protein was biologically functional. Confocal examinations revealed that GFP fluorescence in the transformant G3-1 was visualized at nuclear positions, whereas fluorescence was dispersed throughout the cells in the GFP-only transformant (Fig. 4A). 4,6-diamidino-2-phenylindole (DAPI) staining showed that GFP fluorescence in G3-1 was restricted to the nucleus (Fig. 4B), suggesting that Cos1 is nucleus-localized.

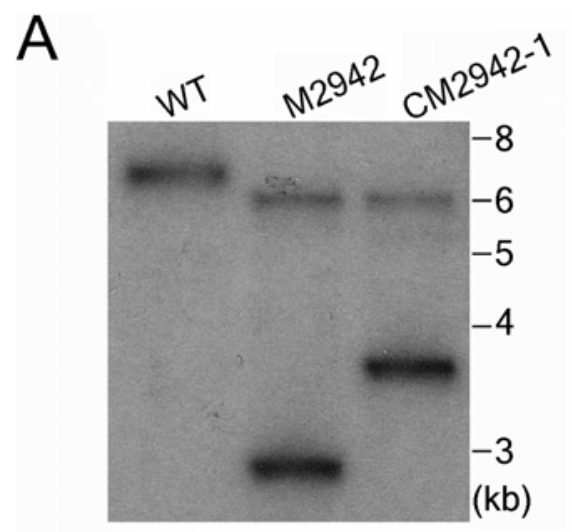

B
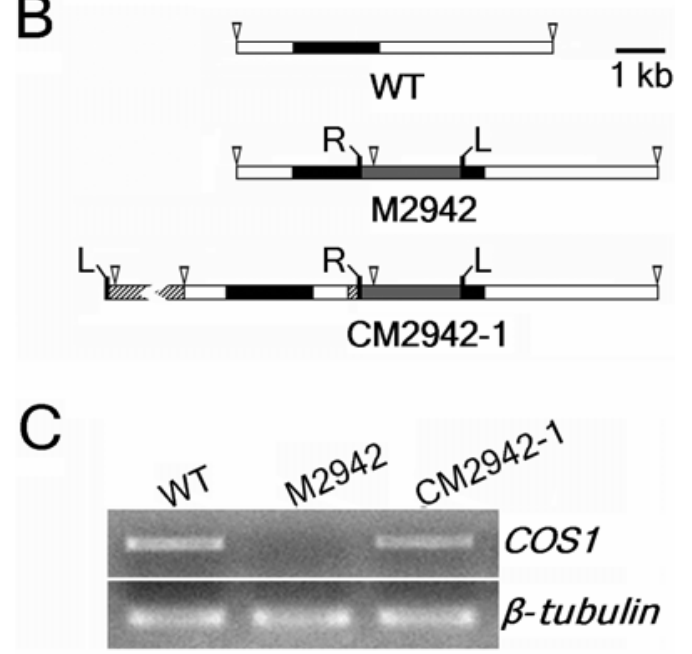

Fig. 3. Molecular analysis and genetic complementation of mutant M2942. A, Southern blot analysis confirming the integration of the complementary construct into M2942 genome. Genomic DNA was digested with KpnI and probed with a full-length coding sequence of conidiophore stalk-less 1 (COS1); WT $=$ wild type. $\mathbf{B}$, Schematic diagrams illustrating T-DNA integrations in complemented strain CM2942-1. Black bars are for COS1 gene, white bars for other genomic DNA, gray bars for $h p t$-containing T-DNA, and striped bars for the T-DNA containing a sulfonylurea-resistant $(S U R)$ gene and the full-length COS1 gene. Triangles mark KpnI sites in the region; $\mathrm{R}$ and $\mathrm{L}$ correspond to T-DNA right border and left border, respectively. The T-DNA of the complementary construct replaced a genomic fragment adjoining the existing T-DNA right border. C, Reverse-transcriptase polymerase chain reaction analysis of transcription of COS1 gene. Primers RT2942F and RT2942R were used for COS1 gene. Primers Tubulin-A and Tubulin-B were used for the $\beta$-tubulin gene as a control.

\section{Mutation of COS1 gene affects transcription} of some developmental homologues.

A conidiophore is a specialized hypha required for conidiogenous cell differentiation (Cole 1986). More conidiophores were observed in the complemented transformant G3-1 than in the wild type (Fig. 5A). Sporulation rates of G3-1 and the wild-type strain were $3.33 \pm 0.94 \times 10^{5}$ conidia $/ \mathrm{cm}^{2}$ and $2.77 \pm$ $0.89 \times 10^{5}$ conidia $/ \mathrm{cm}^{2}$, respectively, demonstrating that $20 \%$ more conidia were produced by G3-1 than by the wild type, though a $t$ test indicated that the difference was not significant. In the complemented transformant G3-1, COS1 was driven by the strong, constitutive $\operatorname{trp} C$ promoter (Hamer and Timberlake 1987), implying that overexpression of COS1 might have promoted differentiation of conidiophores.

A number of regulators required for conidiophore development have been characterized in A. nidulans and N. crassa (Adams et al. 1998; Springer 1993). Genomic sequence analysis revealed nine homologous genes in $M$. oryzae that show high similarity to developmental genes of A. nidulans or $N$. crassa (Table 1). RT-PCR was then carried out to test whether transcription of these nine genes was affected by COS1. The results revealed that $M G G \_02246.5$, a homologue of con- 6 of $N$. crassa, was downregulated in aerial cultures of M2942 and another gene, $M G G \_14517.5$, homologous to flbA of $A$. nidulans, was upregulated in either submerged or aerial cultures of M2942 (Fig. 5B). No significant differences were observed among the other genes. It is noteworthy that transcripts of all homologues were present in aerial cultures, and four of these were exclusively detected in aerial cultures, consistent with the
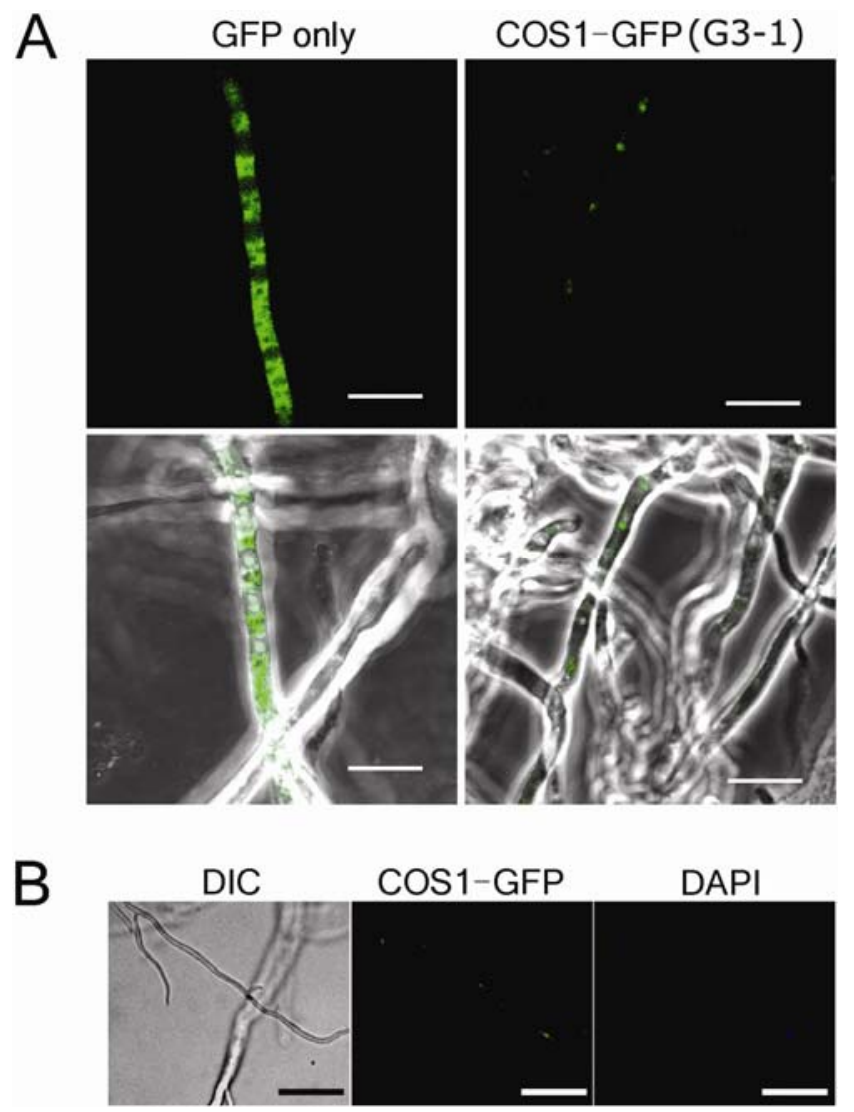

Fig. 4. Subcellular localization of conidiophore stalk-less1-green fluorescent protein (COS1-GFP) fusion protein. A, Confocal micrographs showing subcellular localization of fusion protein COS1-GFP. A transformant expressing GFP only was used as a control. Bar scale $=10 \mu \mathrm{m}$. B, Colocalization of COS-GFP fluorescence and 4,6-diamidino-2-phenylindole (DAPI) stain in strain G3-1. DIC = differential interference contrast. Bar scale = $20 \mu \mathrm{m}$. 
likely function of the gene products in control of sporulation. These results, together with the nuclear localization of Cos1, suggested that Cos1 may function as a transcriptional regulator of genes that are responsible for sporulation in M. oryzae.

Cos1 is not required for pathogenicity but mutation of COS1 may initiate an unknown mechanism of infection.

In general, $M$. oryzae infects rice aerial organs such as leaves and stems via a specialized structure, an appressorium, which develops from a conidium. However, hyphae can also invade rice roots (Sesma and Osbourn 2004) and wounded leaf tissue (Silue et al. 1998). In root infection assays, we observed
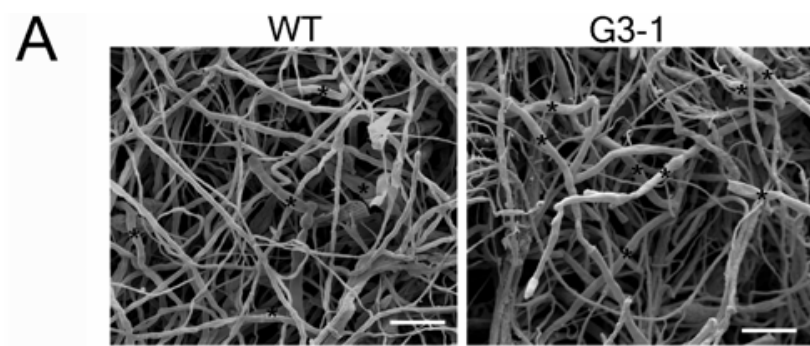

B

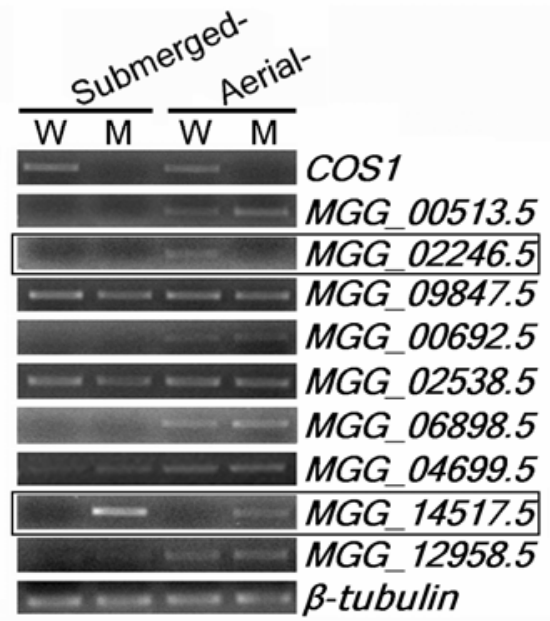

Fig. 5. Effects of conidiophore stalk-less1 (COS1) gene on sporulation and transcription of other genes. A, Scanning electron microscopy photographs showing the difference of conidiophore stalks. Each conidiophore stalk is marked with an asterisk. In this representative field of view, five conidiophore stalks were found for the wild type and eight for G3-1. Scale bar = $20 \mu \mathrm{m}$. B, Reverse-transcriptase polymerase chain reaction-based analysis of transcription of sporulation-related homologous genes. Two homologues affected by the mutation of $\operatorname{COS} 1$ gene are highlighted by rectangles; 30 amplification cycles were for the $\beta$-tubulin gene and 36 cycles for the others. $\mathrm{W}=\mathrm{WT}$ and $\mathrm{M}=\mathrm{M} 2942$. browning and stunted growth of roots inoculated with mycelia of either the wild type or M2942 (Fig. 6A), implying that Cos1 was not required for pathogenicity. This result was reinforced by foliar infection assays, in which mycelia of M2942 caused infectious symptoms on wounded leaves of barley and rice (Fig. 6B).

Surprisingly, however, M2942 appeared to cause more severe symptoms than the wild type (Fig. 6B), suggesting that mycelia of M2942 may be more aggressive. Strikingly, intact (unwounded) rice leaves inoculated with mycelia of M2942 also showed symptoms resembling those caused by conidia, though the size of symptoms was smaller (Fig. 6B). However, mycelia of the wild type did not cause any visible symptoms (Fig. 6B).

To confirm the observation, a COS1 deletion mutant KO-23 was generated by gene replacement (Supplementary Fig. S1). KO-23 also showed a loss-of-sporulation phenotype identical to M2942. In inoculation of intact (unwounded) leaves with mycelia, we found that both M2942 and KO-23 caused visible symptoms resembling those observed in Figure 6C. Moreover, similar phenotypes were observed when mycelia were applied to leaf axils (Fig. 6D). In contrast, the wild type and the rescued strain could not cause infection (Fig. 6C and D).

Additionally, leaf sheaths inoculated with mycelia of M2942 showed browning of host cells where underlying bulbous invading hyphae were observed (Fig. 7A through D). Appressorium-like structures developed at hyphal tips of M2942 on surfaces of leaf sheaths were also observed (Fig. 7E). No similar phenomenon, however, was found in leaf sheaths inoculated with wild-type mycelia. These results suggest that mutation of COS1 may enable an unknown mechanism of mycelial infection of M. oryzae.

\section{DISCUSSION}

Phenotypically, sporulation mutants range from conidiophore-less, conidiophore bearing-no-conidia, to various abnormal conidial shapes. In this study, we identified a sporulation mutant of $M$. oryzae, M2942, which is conidia-less but has a radial growth and colony morphology similar to the wild type when grown on either oatmeal agar or complete medium (CM). This mutant and the deletion mutant KO-23 are markedly deficient in melanin (Fig. 8). Melanin-producing fungi produce melanin in response to environmental stresses (Bell and Wheeler 1986). In our observations, however, the melanin deficiency did not appear to affect the mutant's growth under several stress conditions. Compared with the wild type, the mutant grew normally on CM supplemented with $1 \mathrm{M}$ sorbitol as an osmotic stress, under high temperature stress at $34^{\circ} \mathrm{C}$, where $37^{\circ} \mathrm{C}$ is the temperature at which growth of wild-type

Table 1. Developmentally homologous genes discovered in Magnaporthe oryzae

\begin{tabular}{|c|c|c|c|c|c|}
\hline Gene & Accession no. & Organism & Description, mutant phenotype & Homologue in M. oryzae & Reference \\
\hline brlA & XM_653485 & Aspergillus nidulans & Indeterminate conidiophore stalks. & MGG_12958.5 & Adams et al. 1998 \\
\hline$f l b A$ & XM_658405 & A. nidulans & $\begin{array}{l}\text { Fluffy autolysis as colony matures; required for } \\
\text { initiation of conidiophore development. }\end{array}$ & MGG_14517.5 & Wieser et al. 1994 \\
\hline$f l b C$ & AF083468 & A. nidulans & Fluffy colonies; delayed conidiophore development. & MGG_04699.5 & Wieser et al. 1994 \\
\hline$f l b D$ & U19882 & A. nidulans & Fluffy colonies; delayed conidiophore development. & MGG_06898.5 & Wieser et al. 1994 \\
\hline $\mathrm{flu} G$ & L27817 & A. nidulans & $\begin{array}{l}\text { Timing of induction of asexual sporulation disrupted; } \\
\text { formation of fluffy colonies. }\end{array}$ & MGG_02538.5 & Lee and Adams 1994 \\
\hline stuA & M83569 & A. nidulans & $\begin{array}{l}\text { Spatially deranged conidiophores (shortened } \\
\text { conidiophores). }\end{array}$ & MGG_00692.5 & Adams et al. 1998 \\
\hline $\operatorname{med} A$ & AF080599 & A. nidulans & $\begin{array}{l}\text { Spatially deranged conidiophores (multiple layers of } \\
\text { sterigmata produced). }\end{array}$ & MGG_09847.5 & Adams et al. 1998 \\
\hline con- 6 & L26036 & Neurospora crassa & $\begin{array}{l}\text { Highly expressed during conidiation, but no } \\
\text { observable phenotype when inactivated. }\end{array}$ & MGG_02246.5 & Madi et al. 1994 \\
\hline con-8 & X07040 & N. crassa & $\begin{array}{l}\text { Highly expressed during conidiation, but no } \\
\text { observable phenotype when inactivated. }\end{array}$ & MGG_00513.5 & Madi et al. 1994 \\
\hline
\end{tabular}


$M$. oryzae ceases, and with peroxide as an oxidative stress (data not shown).

We have confirmed that the COS1 gene is a determinant of sporulation or conidiophore development in $M$. oryzae. Cos1 may function as a transcriptional regulator affecting genes required for conidiophore development. First, Cos 1 shares a high similarity at the region of zinc-finger motif with Azf1p, which is characterized as a nuclear-localized suppressor for a mutated mitochondrial transcription apparatus (Bröhl et al. 1994; Stein et al. 1998). By examining GFP-tagged Cos1, we showed that $\operatorname{Cos} 1$ is also a nucleus-localized protein. Second, in RT-PCR-based analysis of expressions of developmental homologues, two genes were found to be affected by mutation of the COS 1 gene. A homologue of con- 6 was downregulated in aerial cultures and a homologue of $f l b A$ was upregulated in either submerged or aerial cultures.

Although functions of the nine homologues tested remain unknown in conidial development of $M$. oryzae, most of these homologues have been characterized in A. nidulans or $N$. crassa. The conidiation-specific gene con- 6 of $N$. crassa is preferentially expressed during the formation of asexual spores, though inactivation of con- 6 by the repeat-induced point mutation process had no demonstrable effect on formation or germination of conidia (White and Yanofsky 1993). Importantly, a common potential regulatory sequence is found within the upstream sequence of the con-6 gene. DNA mobility shift analysis supported that this regulatory sequence bound to a binding factor (White and Yanofsky 1993). These results suggest that other factors may directly regulate the expression of the con- 6 gene during conidiation. flbA mutant strains of $A$. nidulans show a fluffy autolytic phenotype because they fail to make the transition from vegetative growth to conidiophore (Adams et al. 1998). The FlbA protein contains a regulator of G-protein signaling (RGS) domain that is required to antagonize G-protein signaling and thereby allow sporulation (Yu et al. 1996). In A. nidulans, FluG is proposed to function as a prokaryotic glutamine synthetase-related enzyme in production of an extracellular developmental signal that directs asexual sporulation (Lee and Adams 1994). Several developmentspecific genes, including $f l b B, f l b C, f l b D, f l b E, b r l A$, and $f l b A$, are involved in the response to the FluG signal, leading to sporulation.

Liu and associates (2007) reported that the M. oryzae protein Rgs1 negatively regulates the heterotrimeric G-protein cascades that enable eukaryotic cells to perceive and respond to external stimuli. As a result, Rgs1 was found to be a negative regulator of conidiation; that is, $R g s$ l-deletion strains produced more conidia than the wild type whereas Rgs1 overexpression inhibited conidiation. Both Rgs 1 and FlbA of A. nidulans share an RGS domain but each has a distinct role in conidiation. $M G G_{-} 14517.5$, the gene that is upregulated by COS1 mutation, encodes a putative protein containing a DEP_RGS7like domain. $M G G_{-} 14517.5$ and $R g s l$ are actually the same gene (Liu et al. 2007). This may explain why $\cos 1$ mutants fail to produce conidia. To elucidate the relationship, a COS1, $M G G \_14517.5$ double mutant is needed for further work.
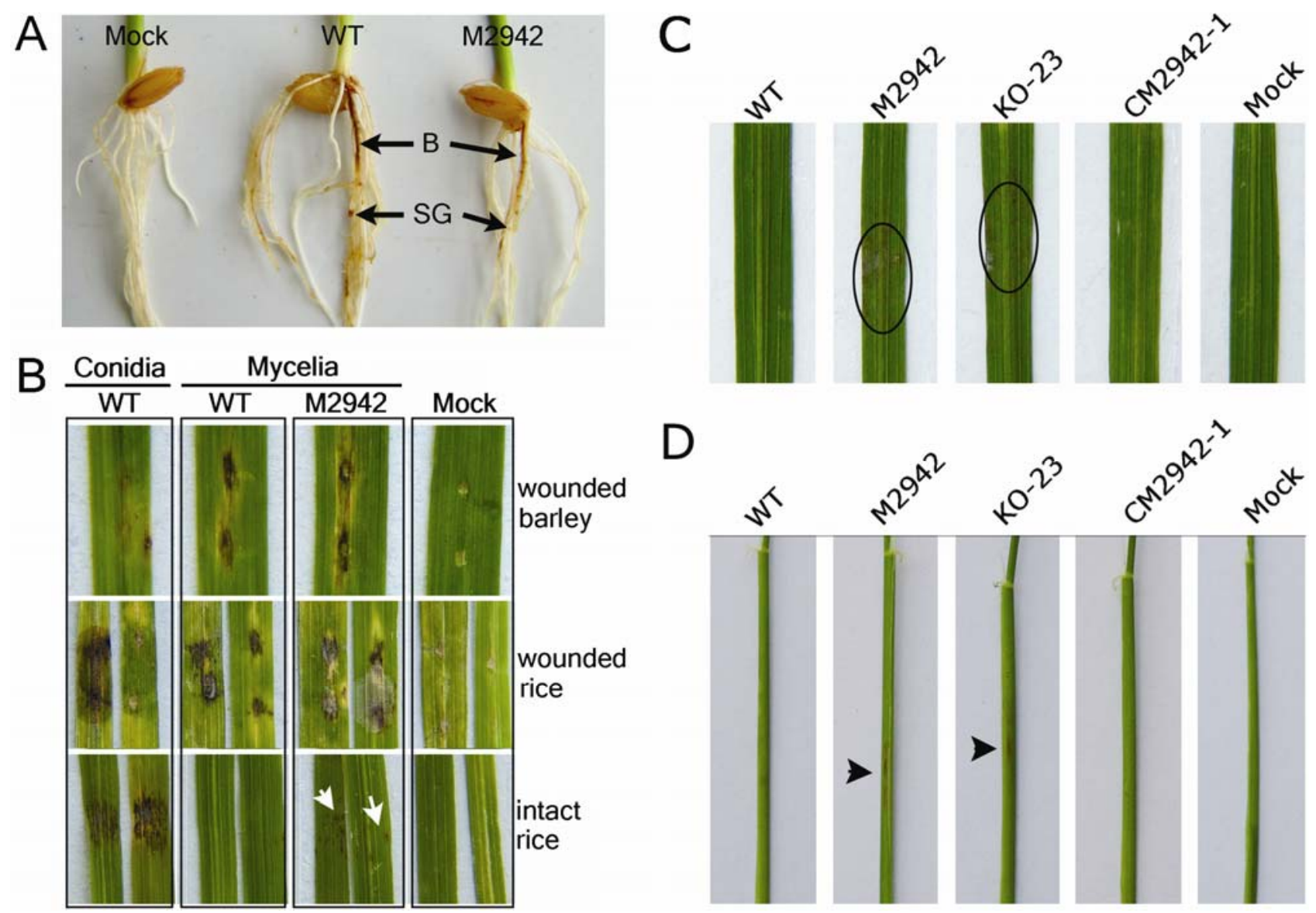

Fig. 6. Mycelial infection assays of Magnaporthe oryzae. A, Roots infected by mycelia of mutant strain M2942 and wild-type (WT) strain Y34. Arrows indicate symptoms characteristic of infected root; B = browning and SG = stunted growth. Uninoculated seedlings (Mock) showed no symptoms. Photographs were taken 12 days after inoculation. B, Inoculation of mycelia on wounded and intact leaves. Arrows point to symptoms caused by M2942 mycelia. C, Inoculation of mycelia on intact leaves and $\mathbf{D}$, inoculation of mycelia on leaf axils. Circles and arrows emphasize the symptoms caused by cos 1 mutants. $\mathbf{B}, \mathbf{C}$, and D, Photographs were taken 4 days after inoculation. 
It was reported that the con 5 mutant displays a similar phenotype to that of M2942, and is characterized by normal radial growth and the absence of conidiophores (Shi and Leung 1995). However, like the wild type, M2942 can produce normal aerial hyphae whereas the con 5 mutant produces only sparse aerial hyphae (Shi and Leung 1995). This suggests that the mutation in M2942 may be different from that of con5. Because the con5 gene has not been characterized, the relationship between M2942 and con5 remains unclear. At this point, the limited knowledge of developmental regulators means that we cannot accurately predict the place of Cos 1 in the conidiogenesis pathway summarized by Shi and associates (1998).

A key finding of this study is that mycelia of M2942 infected susceptible host cells and caused obvious symptoms on leaf blades. The fungus usually depends on an appressorium-mediated mechanism to infect rice leaves, whereby it pierces the tough cuticle barrier and colonizes plant tissue. However, mycelia of M2942 avoid this step in leaf infection. Koga and associates (2004) observed that a cellular resistance reaction occurred even in a susceptible cultivar after inoculation of intact leaf sheaths with conidia. Similarly, we observed frequent browning and death of host cells 3 days after leaf sheath inoculation. More importantly, typical invasive hyphae were found underlying browning cells, and appressorium-like structures were also observed. These appressorium-like structures were similar to those observed by Lee and Dean (1993). In their study, tips of vegetative hyphae could be induced to differentiate into appressoria on hydrophobic surfaces. In our observations, however, those appressorium-like structures could not be induced on a hydrophobic film, even with the presence of cAMP. Presently, we have no adequate evidence to draw a conclusion that these appressorium-like structures are
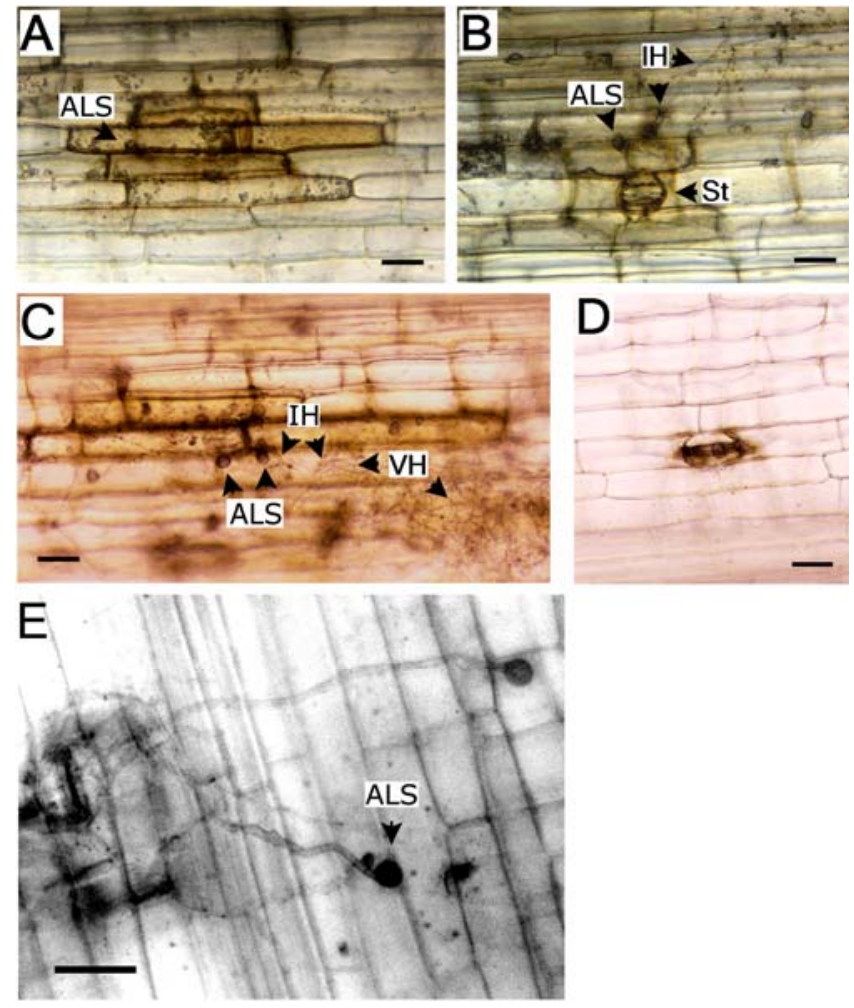

Fig. 7. Leaf sheaths inoculated with mycelia of M2942. A, B, C, and D, Browning or dead cells observed. E, Appressorium-like structures (ALS) developed at tips of hyphae. Bar scale $=50 \mu \mathrm{m}$. No browning or dead cells were found on leaf sheaths inoculated with wild-type mycelia (data not shown). Photographs were taken 3 days after inoculation. IH, invasive hyphae; St, stoma; VH, vegetative hyphae. responsible for the mycelial infection of M2942 though, most likely, they are. Apparently, the appressorium-like structures formed on surfaces of leaf sheath are pigmented, resembling those wild-type appressoria developed on inductive surfaces. Associating with what was observed for plate cultures of M2942 and KO-23, we propose that mutation of the COS1 gene may have an effect on the melanin production for vegetative growth but not for infectious organs.

During root infection, hyphae of the fungus develop structures resembling hyphopodia of root-infecting fungi at infection sites, often with associated infection pegs to penetrate into epidermal cells (Sesma and Osbourn 2004). This provides an example of the diverse strategies that the fungus has developed to infect its host plants. In our study, we provide further evidence of mycelial infection of $M$. oryzae.

Based on our observations, we speculate that stomata on rice leaves may be targets for mycelial attack of M2942, because browning stomata were frequently observed associating or not with browning and death of host cells (Fig. 7B and D). Further research on this topic will improve our understanding of fungal development and provide useful information for new strategies of epidemic control.

In conclusion, our results demonstrated that Cos 1 may be a transcriptional regulator that is essential for conidiophore development by means of up- or downregulating related genes. Mutation of COS1 may aggravate infection of wounded or unwounded leaves. Though COS1 is not required for pathogenicity, mutation of COS1 may trigger an unknown mechanism enabling successful infection by mycelia.

\section{MATERIALS AND METHODS}

\section{Strains and growth conditions.}

A Chinese field strain of $M$. oryzae Y34 was used in this study as the wild type. The strain is a gift from J. Li of the Yunnan Academy of Agricultural Science, China. Other strains used in this study are derivates from strain Y34. Except for cultures with specific requirements, all strains were cultured on oatmeal

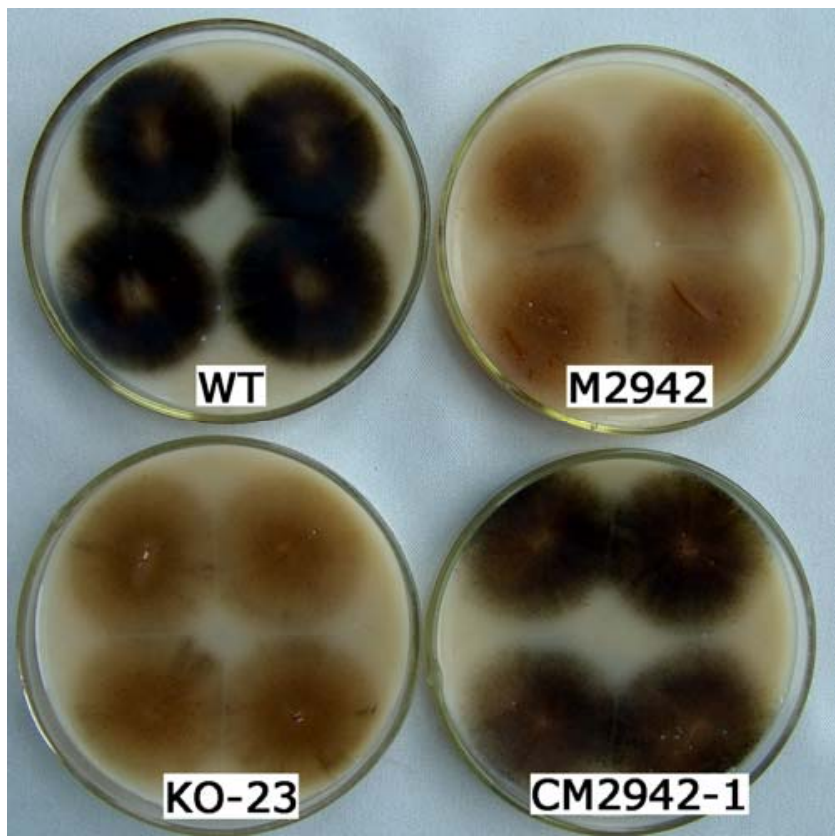

Fig. 8. Morphological phenotypes of the wild type (WT), mutant strains, KO-23, and the complemented strains CM2942-1 on agar plates. The cos 1 mutant M2942 displays melanin deficient. Aerial mycelia grown on oatmeal agar were scraped off to expose pigmented media in 7 days. 
agar plates at $28^{\circ} \mathrm{C}$ under continuous fluorescent light for several days for conidiation or to assess the growth and colony characteristics. Strains were stored on desiccated filter paper at $-20^{\circ} \mathrm{C}$. For DNA and RNA extraction and for leaf inoculation, mycelia were collected by being filtered with one layer of Miracloth (Calbiochem, La Jolla, CA, U.S.A.). The liquid cultures were grown in $\mathrm{CM}(0.6 \%$ yeast extract, $0.6 \%$ tryptone, $1 \%$ sucrose), which were shaken at $180 \mathrm{rpm}$ at $28^{\circ} \mathrm{C}$ for 2 days.

\section{Nucleic acid manipulation.}

Fungal genomic DNA was extracted as previously described (Talbot et al. 1993). For total RNA isolation, submerged mycelia were collected as above, and aerial mycelia were harvested from 7-day-old oatmeal agar plates with a triangular scraper. An acid guanidinium thiocyanate-phenol-chloroform RNA extraction procedure was followed (Siebert and Chenchik 1993). Nucleotide sequencing was performed by Invitrogen Co. (Shanghai, China). We followed standard procedures for manipulations such as plasmid DNA isolation, restriction enzyme digestion, and hybridization (Sambrook et al. 1989).

\section{RT-PCR and TAIL-PCR.}

First-strand cDNA synthesis was carried out with a SuperScript first-strand synthesis system (Invitrogen Co.) using oligo(dT) primers and total RNA from submerged or aerial mycelia as the template. For cloning the coding sequence of the COS1 gene, we designed a pair of primers, UP3977F and DN3977R. The primers theoretically amplify a COS1-containing fragment with additional $67 \mathrm{bp}$ ahead of the start codon and $28 \mathrm{bp}$ following the stop codon. The amplified product was cloned into the pGEM-T Easy vector (Promega Corp., Madison, WI, U.S.A.) giving pT-CS-COS1, which was then sequenced. For detecting transcripts of the nine homologues, the following primers were used: RT2942F and RT2942R (COS1), HbrlA-F and HbrlA-R (MGG_12958.5), HflbA-F and HflbA-R (MGG_14517.5), HflbC-F and HflbC-R (MGG_04699.5), $\mathbf{H} f l b D-F$ and HflbD-R (MGG_06898.5), HfluG-F and HfluG-R (MGG_02538.5), HstuA-F and HstuA$\mathrm{R}$ (MGG_00692.5), HmedA-F and HmedA-R (MGG_09847.5), Hcon6-F and Hcon6-R (MGG_02246.5), Hcon8-F and Hcon8-R (MGG_00513.5), and Tubulin-A and Tubulin-B ( $\beta$-tubulin). TAIL-PCR was performed as described by Li and associates (2007).

\section{Microscopy.}

To stain conidiophores, a tablet of oatmeal agar inoculated with the fungal strain of interest was placed on a sterile glass slide in a plate, kept moist, and grown for 3 days at $28^{\circ} \mathrm{C}$ under continuous fluorescent light. The agar tablet was then removed. A few drops of 1:3 dilution of lactophenol cotton blue stain were mounted and a coverslip was immediately applied to the slide. The lactophenol cotton blue stain was composed of $20.0 \mathrm{~g}$ of phenol, $0.6 \mathrm{~g}$ of cotton blue, $44 \mathrm{ml}$ of glycerol, $16 \mathrm{ml}$ of lactic acid, and $20 \mathrm{ml}$ of deionized water. The slide was examined under a light microscope (Olympus BX51) immediately after staining. GFP was visualized with a confocal microscope (Leica TCS SP2, excitation at $488 \mathrm{~nm}$ and emission at 505 to $530 \mathrm{~nm}$ ). Nuclei were visualized using DAPI (Sigma, Tokyo) at $0.8 \mathrm{mg} / \mathrm{ml}$ and samples were observed and photographed with a fluorescence microscope (Olympus BX51).

\section{Plasmid construction and transformation.}

For the complementation experiment, the plasmid pSULPHC3977 was constructed. A 3.2-kb fragment containing the fulllength COS1 gene was amplified with primers MG3977F and MG3977R and cloned into the pMD18-T vector (Takara,
Tokyo), giving pMD18-C3977. The amplified fragment was then cloned between SalI and EcoRI sites on pMD18-C3977 and ligated to the same sites on pSULPH-GFP (Sesma and Osbourn 2004) in place of the GFP cassette, yielding the plasmid pSULPH-C3977. The plasmid was introduced into M2942 with an Agrobacterium-mediated transformation method as described by Li and associates (2007), yielding the complemented strain CM2942-1. A minor modification was that 2day-old mycelia of M2942 cultured in liquid CM replaced conidia as the recipient. Transformants grown on sulfonylurea at $100 \mu \mathrm{g} / \mathrm{ml}$ were selected for further investigation.

For determining the subcellular localization of $\operatorname{Cos} 1$, the plasmid pCB1532-COS1-GFP was constructed. A 1.5-kb fragment containing the coding sequence of the COS1 gene was amplified with primers FCS3-1 (EcoRI site added) and FCS32 (BglII site added) from pT-CS-COS1. Another 720-bp fragment containing the coding sequence of $s G F P$ was amplified with FCSG-1 (BglII site added) and FCSG-2 (BamHI site added) from pSULPH-GFP. The two fragments were digested with $B g l I I$, then linked together with T4 ligase (NEB) and cloned into the pGEM-T Easy vector, giving pT-COS1-GFP. A 2.2-kb EcoRI-BamHI fragment containing the COS1-GFP sequence on pT-COS1-GFP was then cloned between EcoRI and BamHI sites in the construct pKS-trpC-P-T, which harbors the trpC gene promoter and terminator, resulting in pKS-PTCOS1-GFP. A 3.3-kb SalI-XbaI fragment containing sequences of PtrpC-COS1-GFP-TtrpC on pKS-PT-COS1-GFP was finally cloned between the $S a l \mathrm{I}$ and $\mathrm{XbaI}$ sites of pCB1532 (Sweigard et al. 1997), leading to pCB1532-COS1-GFP (Supplementary Fig. S2). As a control, the plasmid pCB1532-GFP expressing only GFP was constructed. A fragment containing the GFP gene was amplified with primers FCSG-3 (EcoRI site added) and FCSG-2 (BamHI site added), digested with EcoRI and BamHI, and cloned into EcoRI and BamHI sites of pCB1532COS1-GFP, leading to pCB1532-GFP. pCB1532-COS1-GFP and pCB1532-GFP were each introduced into M2942 by plasmid transformation (Shi and Leung 1995), resulting in the strain G3-1 and the GFP-only control strain, respectively. Transformants grown on sulfonylurea at $100 \mu \mathrm{g} / \mathrm{ml}$ were harvested.

For generating a COS1 deletion mutant, a 1.7-kb upstream fragment with primers U1 and U2 (with EcoRI site) and a 900bp downstream fragment with primers D1 (with EcoRI site) and D2 were amplified. Two fragments were linked together with EcoRI and XhoI in the plasmid pT1-Easy (Transgen, Beijing). A 1.4-kb EcoRI-digested fragment containing the modified $h p t$ gene under the $\operatorname{trpC}$ promoter from plasmid pCB1003 (Carroll et al. 1994) was then cloned into the EcoRI site, resulting in plasmid pT1-U5HD2. This plasmid was linearized with $X h o I$ and then introduced into protoplasts of the wild-type strain, resulting in KO-23. Transformants were validated by PCR analysis.

\section{Inoculation of root, leaf, and leaf sheath.}

We used Nipponbare, a rice cultivar susceptible to the fungal strain Y34, and barley cv. Golden Promise to perform inoculation assays. Roots were infected as described by Dufresne and Osbourn (2001). Leaf infection was carried out according to Silue and associates (1998) with modifications. Briefly, young leaves of barley and rice were cut into 4-cm segments and placed on wet filter paper in a plate. Leaf segments were wounded by stabbing with a pipette tip. Mycelia were collected and washed with sterile water four to five times, resuspended in $0.02 \%$ Tween-20 at a ratio of 1:20 (fresh weight), and vortexed vigorously. For the pathogenicity test, leaf segments and leaf axils were inoculated with mycelial suspension and kept moist at $28^{\circ} \mathrm{C}$ under continuous fluorescent light for 3 to 5 days. Wild-type conidia were suspended in 0.02\% Tween-20 
at a concentration of $5 \times 10^{4} / \mathrm{ml}$ and applied onto the leaf segments and leaf axils.

\author{
List of oligonucleotides used in this study. \\ UP3977F: 5'-CTTCTGTCCTCGATCATCGA-3' \\ DN3977R: 5'-CTTCCAGTGAGATGATTTGAG-3' \\ MG3977F: 5'-TCACTCCCAGCAGACAAGG-3' \\ MG3977R: 5'-TTGAATCCCTGTCCGCTC-3' \\ FCS3-1: 5'-CGGAATTCATGAGCACTATGCAACCTCC-3' \\ FCS3-2: 5'-TGAAGATCTGTGACGTTGACCAGCAAAG \\ A-3' \\ FCSG-1: 5'-TGAAGATCTATGGTGAGCAAGGGCGAGG \\ A-3' \\ FCSG-2: 5'-ACGGGATCCTTACTTGTACAGCTCGTCC \\ A-3' \\ FCSG-3: 5'-CGGAATTCATGGTGAGCAAGGGCGAGG \\ A-3'
} RT2942F: 5'-CACGAAAGGCAACCTGCAC-3' RT2942R: 5'-ATCTCGGTGCCAACGTGAC-3' HbrlA-F: 5'-CTCTACGAGCAATTGACCAA-3' HbrlA-R: 5'-ACCCATAGGACCTTGTGAAT-3' HflbA-F: 5'-CCAAAGAAATGGCGAGGT-3' HflbA-R: 5'-CGATCTCAACTGCTTCCCT-3' HflbC-F: 5'-GTCTACGACAGGATACGCAG-3' HflbC-R: 5'-TTAGAGCTGAATTGGCCC-3' HflbD-F: 5'-CCTAGACAAAGCGCATCAC-3' HflbD-R: 5'-ATCTGTATCGGCGCTCAT-3' HfluG-F: 5'-AAGCCTAAGCAAGGTCTCC-3' HfluG-R: 5'-CTTTCCTCATAAATCGGTCG-3' HstuA-F: 5'-TCCTGGAAGTACGCTACAAA-3' HstuA-R: 5'-TGTACAAGTTGCTCGTGGA-3' HmedA-F: 5'-GTACATTTGCGTCTCGTTTG-3' HmedA-R: 5'-CAAGAGCGTCATGCTGTAAC-3' Hcon6-F: 5'-CACAAGGCCAACCTCAA-3' Hcon6-R: 5'-TCTCCATCTCCTCGAGAC-3' Hcon8-F: 5'-GATTCATCCGCAGCAAA-3' Hcon8-R: 5'-CACATCTTGCCAAACAGG-3' Tublin-A: 5'-GTGACCCTCGCAACGGAAAG-3' Tublin-B: 5'-CGACGAACTGGATGCTACGC-3' U1: 5'-GCGAGCGACGTTTGTACTTG-3' U2: 5'-AGAATTCAAAGATCCGCCGCAGGTTA-3' D1: 5'-AGAATTCAGCTTCCCAGCGTGTCAAT-3'

D2: 5'-GAGCGGACAGGGATTCAA-3'

D3: 5'-GTCATCTAGTGGTGGCGTCC-3'

P1: 5'-GCTCTAGACTATGTATGCTGGCT-3'

P2: 5'-ACCGAGAGATTCATCATCTAGAGC-3'

H1: 5'-TGAACTCACCGCTGACGTCGT-3'

H2: 5'-GACAGACGTCGCGGTGAGTT-3'

\section{ACKNOWLEDGMENTS}

We thank W. Zhuang at the Institute of Microbiology, Chinese Academy of Sciences for advice on microscopic examination of conidiophores and for the gift of the lactophenol cotton blue stain. This project was supported by the Ministry of Science and Technology of China (Grant 2006CB101900).

\section{LITERATURE CITED}

Adams, T. H., Wieser, J. K., and Yu, J. H. 1998. Asexual sporulation in Aspergillus nidulans. Microbiol. Mol. Biol. Rev. 62:35-54.

Barr, M. E. 1977. Magnaporthe, Telimenella, and Hyponectria (Physosporellaceae). Mycologia 69:952-966.

Bell, A. A., and Wheeler, M. H. 1986. Biosynthesis and functions of fungal melanins. Annu. Rev. Phytopathol. 24:411-51.

Bröhl, S., Lisowsky, T., Riemen, G., and Michaelis, G. 1994. A new nuclear suppressor system for a mitochondrial RNA polymerase mutant identifies an unusual zinc-finger protein and a polyglutamine domain protein in Saccharomyces cerevisiae. Yeast 10:719-731.
Carroll, A. M., Sweigard, J. A., and Valent, B. 1994. Improved vectors for selecting resistance to hygromycin. Fungal Genet. Newsl. 41:22.

Cole, G. T. 1986. Models of cell differentiation in conidial fungi. Microbiol. Rev. 50:95-132.

Dean, R. A., Talbot, N. J., Ebbole, D. J., Farman, M. L., Mitchell, T. K., Orbach, M., Thon, M., Kulkarni, R., Xu, J. R., Pan, H.., Read, N. D., Lee, Y.H., Carbone, I., Brown, D., Oh, Y.Y., Donofrio, N., Jeong, J.S., Soanes, D. M., Djonovic, S., Kolomiets, E., Rehmeyer, C., Li, W., Harding, M., Kim, S., Lebrun, M. H., Bohnert, H., Coughlan, S., Butler, J., Calvo, S., Ma, L. J., Nicol, R., Purcell, S., Nusbaum, C., Galagan, J. E., and Birren, B.W. 2005. The genome sequence of the rice blast fungus Magnaporthe grisea. Nature 434:980-986.

Dufresne, M., and Osbourn, A. E. 2001. Definition of tissue-specific and general requirements for plant infection in a phytopathogenic fungus. Mol. Plant-Microbe Interact. 14:300-307.

Hamer, J. E., and Timberlake, W. E. 1987. Functional organization of the Aspergillus nidulans trpC promoter. Mol. Cell Biol. 7:2352-2359.

Hamer, J. E., Valent, B., and Chumley, F. G. 1989. Mutations at the SMO genetic locus affect the shape of diverse cell types in the rice blast fungus. Genetics 122:351-361.

Howard, R. J. 1994. Cell biology of pathogenesis. Pages 3-22 in: Rice Blast Disease. R. S. Zeigler, S. A. Leong, and P. S. Teng, eds. CAB International Press, Wallington, Oxon, U.K.

Iuchi, S. 2001. Three classes of $\mathrm{C}_{2} \mathrm{H}_{2}$ zinc finger proteins. Cell. Mol. Life Sci. 58:625-635.

Koga, H., Dohi, K., Nakayachi, O., and Mori, M. 2004. A novel inoculation method of Magnaporthe grisea for cytological observation of the infection process using intact leaf sheaths of rice plants. Physiol. Mol. Plant Pathol. 64:67-72.

Lau, G. W., and Hamer, J. E. 1998. Acropetal: a genetic locus required for conidiophore architecture and pathogenicity in the rice blast fungus. Fungal Genet. Biol. 24:228-239.

Lee, B. N., and Adams, T. H. 1994. The Aspergillus nidulans fluG gene is required for production of an extracellular developmental signal. Genes Dev. 8:641-651.

Lee, Y.-H., and Dean, R. A. 1993. cAMP regulates infection structure formation in the plant pathogenic fungus Magnaporthe grisea. Plant Cell 5:693-700.

Li, G., Zhou, Z., Liu, G., Zheng, F., and He, C. 2007. Characterization of T-DNA insertion patterns in the genome of rice blast fungus Magnaporthe oryzae. Curr. Genet. 51:233-243.

Liu, H., Suresh, A., Willard, F. S., Siderovski, D. P., Lu, S., and Naqvi, N. I. 2007. Rgs1 regulates multiple $\mathrm{G} \alpha$ subunits in Magnaporthe pathogenesis, asexual growth and thigmotropism. EMBO (Eur. Mol. Biol. Organ.) J. 26:690-700.

Madi, L., Ebbole, D. J., White, B. T., and Yanofsky, C. 1994. Mutants of Neurospora crassa that alter gene expression and conidia development. Proc. Natl. Acad. Sci. U.S.A. 91:6226-6230.

Mullins, E. D., Chen, X., Romaine P., Raina, R., Geiser, D. M., and Kang, S. 2001. Agrobacterium-Mediated Transformation of Fusarium oxysporum: an efficient tool for insertional mutagenesis and gene transfer. Phytopathology 91:173-180.

Odenbach, D., Breth, B., Thines, E., Weber, R. W. S., Anke, H., and Foster, A. J. 2007. The transcription factor Con7p is a central regulator of infection-related morphogenesis in the rice blast fungus Magnaporthe grisea. Mol. Microbiol. 64:293-307.

Sambrook, J., Fritsch, E. F., and Maniatis, T. 1989. Molecular Cloning. Cold Spring Harbor Laboratory Press, Cold Spring Harbor, NY, U.S.A.

Sesma, A., and Osbourn, A. E. 2004. The rice leaf blast pathogen undergoes developmental processes typical of root-infecting fungi. Nature 431:582-586.

Shi, Z., and Leung, H. 1994. Genetic analysis and rapid mapping of a sporulation mutation in Magnaporthe grisea. Mol. Plant-Microbe Interact. $7: 113-120$.

Shi, Z., and Leung, H. 1995. Genetic analysis of sporulation in Magnaporthe grisea by chemical and insertional mutagenesis. Mol. PlantMicrobe Interact. 8:949-959.

Shi, Z., Christian, D., and Leung, H. 1998. Interactions between spore morphogenetic mutations affect cell types, sporulation, and pathogenesis in Magnaporthe grisea. Mol. Plant-Microbe Interact. 11:199-207.

Siebert, P. D., and Chenchik, A. 1993. Modified acid guanidinium thiocyanate-phenol-chloroform RNA extraction method which greatly reduces DNA contamination. Nucleic Acids Res. 21:2019-2020.

Silue, D., Tharreau, D., Talbot, N. J., Clergeot, P. H., Notteghem, J. L., and Lebrun, M. H. 1998. Identification and characterization of $\operatorname{apfl}^{-}$in a non-pathogenic mutant of the rice blast fungus Magnaporthe grisea which is unable to differentiate appressoria. Physiol. Mol. Plant Pathol. 53:239-251.

Springer, M. L. 1993. Genetic control of fungal differentiation: the three sporulation pathways of Neurospora crassa. BioEssays 15:365-374. 
Stein, T., Kricke, J., Becher, D., and Lisowsky, T. 1998. Azf1p is a nuclearlocalized zinc-finger protein that is preferentially expressed under nonfermentative growth conditions in Saccharomyces cerevisiae. Curr. Genet. 4:287-296.

Sweigard, J., Chumley, F., Carroll, A., Farrall, L., and Valent, B. 1997. A series of vectors for fungal transformation. Fungal Genet. Newsl. 44:52-53.

Talbot, N. J. 2003. On the trail of a cereal killer: exploring the biology of Magnaporthe grisea. Annu. Rev. Microbiol. 57:177-202.

Talbot, N. J., Salch, Y. P., Ma, M., and Hamer, J. E. 1993. Karyotypic variation within clonal lineages of the rice blast fungus, Magnaporthe grisea. Appl. Environ. Microbiol. 59:585-593.

Teng, P. S., Klein-Gebbinck, H. W., and Pinnschmidt, H. 1991. An analysis of the blast pathosystem to guide modeling forecasting. Pages 1-30 in: Rice Blast Modeling and Forecasting, International Rice Research Institute, Los Banos, Philippines.

Valent, B., and Chumley, F. G. 1991. Molecular genetic analysis of the rice blast fungus Magnaporthe grisea. Annu. Rev. Phytopathol. 29:443-467. White, B. T., and Yanofsky C. 1993. Structural characterization and ex pression analysis of the Neurospora conidiation gene con-6. Dev. Biol. 160:254-264.

Wieser, J., Lee, B. N., Fondon, J. W., and Adams, T. H. 1994. Genetic requirements for initiating asexual development in Aspergillus nidulans. Curr. Genet. 27:62-69.

Yu, J. H., Wieser, J., and Adams, T. H. 1996. The Aspergillus FlbA RGS domain protein antagonizes $\mathrm{G}$ protein signaling to block proliferation and allow development. EMBO (Eur. Mol. Biol. Organ.) J. 15:51845190 .

\section{AUTHOR-RECOMMENDED INTERNET RESOURCE}

Broad Institute's Magnaporthe grisea database: www.broad.mit.edu/annotation/fungi/magnaporthe 